\title{
Nutrição Estética: valorização do corpo e da beleza
} através do cuidado nutricional

\author{
Esthetic nutrition: body and beauty enhancement \\ through nutritional care
}

Juliana da Silveira Gonçalves Zanini W itt ${ }^{1}$

Aline Petter Schneider ${ }^{2}$

\footnotetext{
${ }^{1}$ Instituto de Pesquisa Ensino e Gestão em

Saúde. Rua Pedro Ivo 891, M ont'Serrat. 90450-210 Porto Alegre RS.

julianas_goncalves@ ibest.com.br

${ }^{2}$ Depto. de M edicina Social FAMED/UFRGS
}

Abstract N owadays, there is an increasing quest for beauty and the models proposed by fashion goods and service segments, to achi eve the perfect body. The standard of beauty corresponds to a thin body, without considering health aspects. The number of women who go on diets to control weight is increasing; and taking this into consideration the objective of this study is to conduct a bibliographical review and extract data on esthetics and body image to support the practice of nutritional care. Socio-cultural aspects, which motivate the quest for the perfect body, as well as body, beauty, esthetics, nutritional counseling and cognitive behavior therapy were examined in this survey. On the basis of this work, it is possible to conclude that the continuing obsession with the body may lead the person to go on diets and other drastic methods to control weight, such as surgical procedures. In this respect, nutritional care is far more than merely recommending a standard diet or giving information, as it represents providing an effective model for nutritional reeducation, prioritizing improvement in the style and quality of life. This article provides data about enhancing esthetics and beauty by means of appropriate nutrition.

Key words Beauty, Body, Diet, Esthetics, Nutritional counseling, Esthetic nutrition
Resumo Atualmente, observa-se um crescimento da busca pela beleza e dos modelos propostos pelos segmentos da moda, de bense serviços em torno do corpo perfeito. 0 modelo de beleza corresponde a um corpo magro, sem considerar aspectos relacionados à saúde. 0 número de mulheres que se submetem a dietas para o controle de peso vem aumentando cada vez mais, e diante de tais conside rações o presente estudo tem por objetivo realizar uma revisão bibliográfica e abstrair elementos sobre estética e imagem corporal para subsidiar a prática da atenção nutricional. Para a revisão foram abordados aspectos socioculturais que incentivam uma busca pelo corpo perfeito, bem como 0 corpo, a beleza, a estética, o aconselhamento nutricional ea terapia cognitiva comportamental. A partir deste trabalho é possível concluir que a preocupação contínua com o corpo pode levar a die tasea outros métodosdrásticos de controlede peso, bem como de procedimentos cirúrgicos. N este sentido, 0 atendimento nutricional émais do que fornecer um padrão de dieta ou informação, repre senta a atuação de um efetivo modelo de reeducação alimentar, priorizando uma melhora no estilo e na qualidade de vida. 0 presente artigo traz dados sobrea valorização estética eda beleza, através da nutrição.

Palavras-chave Beleza, Corpo, Dieta, Estética, Aconsel hamento nutricional, N utrição estética 
Introdução

Viver com saúde e boa forma é uma das preocupações que perpassa todos os segmentos da sociedade, principalmenteo do público feminino.

As demarcações de um corpo bonito traduzem insígnias de uma nova ordem que se instaurou e que ganha destaque neste início de século: corpos fortes, torneados, magros e perfeitos. A sociedade de consumo atual não os exime das exigências quanto ao padrão de beleza que impõe, desconsiderando as inúmeras desigualdades e diversidades existentes.

A preocupação com o corpo saudável, e acima de tudo bonito atravessa contemporaneamente, os diferentes gêner os, faixas etárias e classes sociais.

Atualmente observa-se um crescimento da busca pela beleza e dos modelos propostos pelos segmentos da moda, de bens e serviços em torno do corpo perfeito. 0 modelo de beleza corresponde a um corpo magro, sem considerar aspectos relacionados à saúde. A imagem corporal parece ser uma marca feminina, onde o número de mulheres que se submetem a dietas para o controle de peso vem aumentando gradativamente.

0 padrão de beleza de corpo magro é veiculado a mensagens de sucesso, controle, aceitação efelicidade. Assim, mulheres acreditam quesendo magras, poderão alcançar todos os seus obje tivos, sendo a perda de peso a solução para todos os seus problemas.

Entretanto, este padrão imposto como o ideal não respeita os diversos biótipos existentes e induz mulheres a se sentirem feias e a desejarem 0 emagrecimento. Para isso, diversas mulheres caem na armadilha da dieta e aderem práticas inadequadas, como o uso de remédios, laxantes, jejum prolongado, excesso de atividade física, entre outros métodos sem se preocupar com os danos que podem causar a sua saúde.

Diante de tais considerações o presente estudo tem por objetivo realizar uma revisão bibliográfica e avaliar a valorização da imagem corporal através do cuidado nutricional subsidiado pela prática da atenção nutricional. Para tal, foram selecionados os seguintes bancos de dados: SCIELO, LILACS e MEDLINE. Foram pesquisados osidiomas: português, inglês eespanhol edefinidos os seguintes descritores: beleza, corpo, esté tica, aconselhamento nutricional, nutrição esté tica e alimentação saudável. Os levantamentos dos estudos referentes ao tema escolhido priorizaram o período de 2000 a 2008, para que a pesquisa contasse com dados mais recentes, não excluindo publicações de datas anteriores, que possuíssem material pertinente ao estudo e que fossem atuais ainda hoje. Além disso, foram pesquisados livros técnicos, sites de internet e revistas cientificas relacionados ao tema principal do estudo.

\section{Beleza, Corpo e Estética}

A imagem corporal é a maneira pela qual o corpo se apresenta para si próprio. A indústria cultural, pelos meios de comunicação, encarre ga-se de criar desejos er eforçar a imagem padronizando corpos ${ }^{1}$.

0 hábito de fazer dietas e de consumir produtos dietéticos são uma das preocupações mais marcantes do público feminino, embora demonstrem uma preocupação excessiva com a quantidade de gordura no corpo, elas evitam comidas que "engordam" e expressão o desejo de serem cada vez mais magras ${ }^{2}$.

A importância dada à imagem, aparência, corpo, beleza e estética é notória nos dias atuais, ondeo culto ao corpo e ao belo épredominante?.

A sociedade contemporânea assiste deslumbrada à passagem dos corpos perfeitos, que invadem progressivamente todos os espaços da vida moderna ${ }^{4}$.

Vivemos em um mundo em que os meios de comunicação veiculam ou produzem notícias, representações eexpectativas nos indivíduos com propagandas, informações e noticiários. Sendo a televisão o veículo de comunicação mais utilizado para o entretenimento e para a educação, representa a maior fonte de informação sobre 0 mundo, sendo capaz de transmitir aos mais diversos lugares e culturas dados sobre como as pessoas se comportam, o que vestem, o que pensam, o que comem e o padrão de beleza ${ }^{5,6}$. Os meios de comunicação padronizam corpos, corpos que se vêem fora de medidas sentem-se cobrados einsatisfeitos. O reforço dado pela mídia em mostrar corpos atraentes, faz com que uma parte de nossa sociedade se lance na busca de uma aparência física idealizada ${ }^{1}$.

Durante muito tempo, temas como beleza, corpo e estética foram considerados menores no campo das ciências sociais brasileiras. Apesar de alguns autores terem tratado destes temas, já no início do século XX, estes demoraram a surgir em nosso país. No entanto, no final do século $X X$ e início do século $X X I$, parece ter ocorrido uma verdadeira explosão de trabalhos científicos, em diferentes áreas de conhecimento, que trazem como objetivo central discutir a singula- 
ridade do corpo, da construção da beleza e esté tica na nossa cultura?.

Pode-se afirmar que o final do século $X X$ e 0 início do século XXI serão lembrados como o momento em que o culto ao corpo se tornou uma verdadeira obsessão. E a associação corpo e prestígio se tornou um elemento fundamental da nossa cultura brasileira. A busca por um modelo ideal de beleza, nunca foi tão estimulado e valorizado ${ }^{7,8}$.

Ao longo dos séculos, os padrões de beleza mudaram. Nos anos 80, ocorreu um crescimento considerável do mercado relacionado à manutenção do corpo. N as últimas décadas, além de ter se estabelecido um fascínio pela imagem, que é medida pelo culto ao corpo, passa a ter um espaço privilegiado na publicidade 9 .

0 aumento da exigência por um padrão de beleza esguio pode ocasionar no indivíduo uma relação entre seu estado nutricional, com uma visão não condizente, e a autopercepção da sua imagem corporal, com al gum sentimento de insatisfação ${ }^{10}$. A idealização da magreza pode influenciar o comportamento das pessoas a fazerem dietas que prometem rápida perda de peso e sem sofrimento ${ }^{11}$.

A mídia veicula um modelo de beleza, que é possível apenas para uma parcela da população mundial, já que para conseguir estes padrões envolve custos para diferentes classes sócias e culturais ${ }^{12}$. Além disso, as propagandas existentes, principalmente nas revistas, de uma forma indireta, afirmam que a aparência física é responsável pela felicidade esucesso, formando uma ilusão de bem-estar que para ser conquistado, será necessário que a pessoa se enquadre no padrão de beleza estabelecida ${ }^{13}$.

A cultura ocidental enfatiza mais a boa forma e a imagem corporal, o que facilita a identificação de incômodos com pequenos excessos de peso. N ossos padrões culturais fazem com que até indivíduos com o peso dentro dos parâmetros da normalidade, possam sentir-se com seu peso acima do desejado ${ }^{14}$.

Segundo Kawalski e Ferreira ${ }^{15}$ o corpo ocidental está em plena metamorfose. Não se trata mais de aceitar como eleé, mas sim decorrigi-lo, transformá-lo e reconstruí-lo. 0 indivíduo busca em seu corpo uma verdade sobre si mesmo que a sociedade não conseguia mais Ihe proporcionar. Sendo que os padrões valorizados não são os mesmos para todos ${ }^{16}$.

A expectativa de corpo das pessoas em relação a esses padrões de beleza é o que provavelmente interliga uma variedade de fenômenos cada vez mais comuns, como a maior incidência de anorexia e bulimia nervosa4. Saikali et al. ${ }^{17}$, descreve anorexia e bulimia como um transtorno caracterizado por um padrão de comportamento alimentar gravemente perturbado, com um controle patológico do peso e por distúrbios da percepção do formato corporal. Para Goldenberg ${ }^{18}$, estes distúrbios deixaram a condição de patologia para a categoria de estilo de vida.

Diante desta preocupação com o corpo, a imagem corporal se torna um tema de interesse da nossa sociedade, sendo tão preponderanteque leva as pessoas a se preocuparem excessivamente com el $a^{3,19}$. D esse ponto de vista, a noção deestética nos assola, já que, sobretudo na sociedade atual, ela é determinada por padrões culturais ${ }^{20}$ que variará de acordo com cada concepção de mundo, fazendo com que haja um corpo típico para cada sociedade. É esse corpo que pode variar de acordo com o contexto histórico ecultural, é o corpo que entra e sai da moda $a^{7,18}$.

O estilo devida contemporâneo evidenciauma necessidade crescente dosindivíduos procurarem formas de se manterem nos padrões estéticos exigidos pela cultura em que, no que se refere ao corpo, prevalecem processos simbólicos funcionais a um mercado controlado por setores hege mônicos que ditem a maneira de ser aceito ${ }^{21}$.

Dentre os fenômenos da procura pela beleza, o crescimento da cirurgia plástica estética merece destaque pelo impacto que as alterações corporais, propostas pela medicina da beleza, causam em relação à imagem corporal ${ }^{5}$.

Segundo a sociedade brasileira de cirurgia plástica, o brasileiro, especialmente a mulher, se tornou logo após o norte-americano, o povo que mais faz plástica no mundo. A lipoaspiração é a cirurgia mais realizada, seguida da operação das mamas, abdômen, pál pebras e nariz. Dados recentes demonstram que a mulher brasileira é campeã na busca de um corpo perfeito ${ }^{18}$.

Diversos estudos atuais estão evidenciando proporções elevadas de comportamentosalimentares considerados anormais ${ }^{22}$ e de insatisfação com a imagem corporal em ambos os sexos, em diferentes faixas etárias e classes sociais ${ }^{23} 0$ que faz aumentar significativamente a procura por profissionais que possam auxiliar na busca por um corpo ideal. São profissionais da área da saúde, como: médicos, cirurgiões plásticos, psicólogos, personal training e nutricionistas.

\section{A nutrição e a dietética}

A alimentação é uma necessidade humana vital, na qual a comida e o ato de se alimentar 
representam diferentes elementos com conotações simbólicas.

A alimentação humana envolve aspectos psicológicos, fisiológicos esocioculturais, setornando um fenômeno de grande complexidade ${ }^{24}$. A comunicação dos conceitos existentes no código da alimentação enutrição deve ser capaz de prover as condições necessárias às mudanças de comportamento no ato de comer ${ }^{25}$.

A ciência da nutrição destaca quetodo indivíduo deveter uma alimentação saudável e equilibrada, tanto em qualidade como em quantidade. Hábitos alimentares inadequados, alto consumo energético e fal has no metabolismo levam à obesidade eà deficiência de nutrientes, que afe tam mais de dois bilhões de pessoas mundialmente. Isto corre porque há um consumo cada vez maior de alimentos industrializados ${ }^{26}$.

A demanda por orientação alimentar tem crescido significativamente. Embora não haja registro sobre seu incremento e sua dimensão, observa-se que tem aumentado tanto na rede básica de saúde, quanto em clínicas e consultórios ${ }^{27}$.

Sabe-se que uma exposição de apenas 30 se gundos a comerciais de alimentos é capaz de influenciar a escolha de um determinado produto, o que mostra que o papel da televisão (TV), no estabelecimento de hábitos alimentares, deve ser investigado. Diante da TV, um indivíduo pode aprender concepçõesincorretas sobreo queéum alimento saudável ${ }^{28}$.

0 profissional da área da saúde e nutrição não pode estar al heio ao que se passa no mercado midiático. De nada adianta prescrever dietas, divulgar práticas alimentares saudáveis, descontextualizadas da forte influência que o público recebe da mídia ${ }^{5}$.

A relação com o corpo, assim como hábitos e práticas alimentares, também são construídos com base em determinações socioculturais, a mídia exerce um papel importante na construção e desconstrução de procedimentos alimentares e padrões de estética. Assim, discursos sobre práticas alimentares para emagrecimento, formam um padrão de corpo ideal, que são transmitidos re presentando interesse de algum segmento. Podemos dizer que são vendidos o tempo todo produtos e serviços que colaboram tanto com a obe sidade, como também com um padrão estético perfeito, construindo um parado $\mathrm{x}^{3,5}$.

Considerando que hábitos alimentares estão inseridos em estruturas culturais, econômicos e políticos, é necessário haver uma maior ênfase na promoção de ações que subsidiem a produção de alimentos saudávei ${ }^{29}$. Não parece haver dúvidas sobrea importância da educação al imentar e nutricional na promoção de práticas alimentares saudáveis. No entanto, as reflexões sobre suas possibilidades e limites, como o modo que ela é concebida, ainda são escassas ${ }^{30}$.

Muitos nutricionistas para promover práticas alimentares saudáveis, utilizam guias como ferramenta de orientação à população que visam à promoção da saúde por meio da formação de hábitos adequados. Um dos motivos para sua utilização éprevenir excessos ecarências nutricionais, uma vez que a essencialidade de sua mensagem é a moderação e a proporcionalidade ${ }^{31}$.

Para M ota et al .32, a criação de um índicecom base em propostas específicas para a população brasileira, equeavaliedeforma qualitativa equantitativa a dieta habitual, faz-se necessária para propor interven ções dietéticas mais eficazes.

A relação alimentação e atividade física com a saúde são estudadas há muitos anos, sendo que os resultados confirmam que a prática de atividadefísica regular euma alimentação equilibrada, atuam diretamentena prevenção das doenças crônicas não transmissíveis e uma vida mais saudável33, além de auxiliar pessoas na busca de seus ideais, como por exemplo, o corpo perfeito.

Para a ciência da nutrição não devemos privar os indivíduos de comer aquilo que se gosta, pois sabemos que 0 ato de comer também é um ato de prazer. Considerando que a proibição não éo melhor caminho, poisa pessoa se sentiráfrustrada e, por conseguinte, abandonará a dieta, 0 ideal éescolher junto ao clienteos alimentos que mais Ihe apetecem e encontrar formas ou técnicas de preparo que os tornem mais saborosos e nutritivos ${ }^{5}$.

Uma alimentação equilibrada quantitativa e qualitativamente, aliada a uma prática esportiva, garante o trabalho metabólico normal, que conduz o indivíduo a atingir e a manter seu peso ideal ou a buscar pelo corpo idealizado. Enfim, uma alimentação saudável e equilibrada dispensa o uso de medicamentos, de dietas milagrosas ou de cirurgias plásticas para conquistar o padrão de beleza desejado5.

\section{Aconselhamento Nutricional}

A procura por aconselhamento nutricional tem aumentado muito nos últimos anos, já que é comprovado que uma boa alimentação pode evitar uma série de doenças, além de trazer inúmeros benefícios a nossa saúde, e uma melhor qualidade de vida. A educação nutricional pode promover o desenvolvimento da capacidade de 
compreender práticas e comportamentos, os conhecimentos ou as aptidões resultantes desse processo contribuem para a integração do indivíduo com o meio social ${ }^{34}$.

O nutricionista deve estar preparado para captar o estado emocional do cliente (ansiedade, nervosismo einsatisfação) declarado verbalmente ou por meio de gestos, postura, movimento do corpo, expressões faciais, qualidade da voz e 0 silêncio. 0 profissional deve, antes de tudo, saber ouvir e saber aceitar, criando um ambiente favorável para a construção de estratégias que favoreçam o desenvolvimento de ações pelo cliente $^{27}$. Considera-se que a ajuda para resolver problemas al imentares insere-se numa busca de âmbito maior por qualidade de vida. Esta ocorre em determinado momento crítico, no qual surge um problema, que, por sua vez, também é fruto de uma história de vida 35 .

A educação ou aconsel hamento nutricional é o processo pelo qual os clientes são efetivamente auxiliados a selecionar ea implementar comportamentos desejáveis de nutrição e estilo de vida. Então, o resultado desse processo não é somente a mel hora do conhecimento sobre nutrição, mas uma mudança de comportamento. A qual deve ser específica às necessidades eà situação de cada indivíduo. 0 consel heiro nutricional éapenasum facilitador das mudanças de comportamento. Ele dá apoio emocional, auxilia na identificação de problemas nutricionais e estilo de vida, sugere comportamentos a serem modificados efacilita a compreensão e o controle do cliente ${ }^{36}$. Conceitua-se 0 aconsel hamento dietético como uma abordagem de educação nutricional, efetuada por meio de diálogo entreo clientee o nutricionista ${ }^{27}$.

É importante considerar que o processo de aconselhamento não busca impor ao cliente respostas prontas para o problema, mas sim estabelecer uma relação de ajuda entre o aconselhador e o cliente, efetivada por meio das etapas do aconselhamento ${ }^{27}$.

As necessidades psicológicas exercem mais influência nos hábitos alimentares do que a lógica. Fornecer informações, prover materiais educativos, criar estatísticas e pesquisas científicas não necessariamente conduzem a uma mudança decomportamento. 0 principal éaprender a entender o cliente e seus problemas ou insatisfações, a ponto de conhecer os obstáculos que ele enfrenta quando tenta alcançar seus objetivos ${ }^{36}$.

0 método mais utilizado por psicólogos é a terapia cognitiva comportamental (TCC), uma intervenção semiestruturada, objetiva e orientada para metas, que aborda fatores cognitivos, emocionais e comportamentais no tratamento dos transtornos de imagem. A TCC ocupa-se da identificação e correção das condições que favorecem o desenvolvimento e a manutenção das alterações cognitivas e comportamentais que caracterizam os casos clíni $\cos ^{37}$.

Consequentemente, a modificação de padrões distorcidos de raciocínio e a reestruturação de crenças supervalorizadas, associadas ao peso e à imagem corporal são focos primários do tratamento, sendo utilizadas várias técnicas cognitivas com essa finalidade. Nos últimos anos os casos mais comuns para a abordagem da TCC foram: os transtornos alimentares e distorção de imagem ${ }^{38}$

Além das técnicas cognitivas, a TCC também emprega as comportamentais para ajudar na modificação dos hábitos alimentares. Diferentes estudos vêm mostrando a eficácia de se associar orientação nutricional eatividadefísica à TCC. A terapia cognitiva pode ser aplicada em pacientes de diversas idades e de diferentes níveis educacionais, econômicos ou culturais, podendo ser realizada individualmente ou em grupos ${ }^{38}$.

Os programas de TCC resultam em melhoras significativas. As quais também são observadas na autoestima, na solução das dificuldades interpessoais, no humor e na qualidade de vida, além de um aumento do sentimento subjetivo de bem-estar. Entretanto, nem todos os pacientes apresentam boa resposta à TCC ${ }^{39}$.

0 avanço na compreensão da natureza humana, além do conhecimento da ciência da nutrição, tem elevado o nível da prática da educação. A experiência tem-nos mostrado que entregar listas de al imentos e dietas calculadas em detalhes não necessariamente garante uma adesão ou motivam os clientes a mudarem seus comportamentos. Não é tão simples assim, pois as pessoas estão ligadas a seus hábitos. A natureza humana, o querer do indivíduo ea saúde mental podem ter um papel significativo no processo de mudança. 0 cliente tem o direito e a responsabilidade de fazer as escolhas sobre seus cuidados com a saúde. 0 papel do educador éfacilitar esse processo, sempre com o objetivo de promover a melhor qualidade de vida ${ }^{37}$.

Além disso, faz-se importante que os profissionais envolvidos, principalmente os das áreas de saúde e educação, atentem cada vez mais para a importância de incentivar as pessoas a conhecerem melhor a si mesmo e ao seu corpo e a refletirem sobre os padrões corporais em voga e a compreenderem essa relação com a sua saúdee qualidade de vida ${ }^{40}$. 


\section{Considerações finais}

Percebe-se quea preocupação com a beleza éalvo das atenções de toda humanidade, e o cuidado estético tem se expandido a cada dia. Esse processo tem um impacto negativo sobre a autoimagem, principalmente das mulheres que se sentem obrigadas a terem um corpo magro. A pressão cultural para emagrecer é um elemento fundamental da etiologia dos transtornos alimentares, que interage com fatores biológicos, psicológicos e familiares. Contudo, é importante mencionar queas alterações em relação à estética eà imagem corporal estão em constante evolução, necessitando assim de uma maior atenção nutricional.

0 atendimento nutricional émais do quefornecer uma informação, o nutricionista atua na reeducação alimentar priorizando uma melhora no estilo de vida, assim cuidar da alimentação é cuidar da vida. Para o profissional que atua diretamente com esta clientela, é de fundamental importância estabelecer juntos os objetivos possíveis de serem alcançados.

Portanto, a nutrição vai além das dietas para perda de peso, o nutricionista atua na promoção da saúde buscando bons hábitos alimentares para uma melhor qualidade de vida, advertindo sobre as crenças e as condutas equivocadas a respeito da alimentação.

Ressaltamos a importância que tem a dimensão educativa dos nutricionistas e profissionais da área da saúde para a população em geral, particularmente para as mulheres. Orientar e informar sobre hábitos alimentares é um desafio que seimpõeàqueles queacreditam que o corpo pode e deve ser visto em suas diferentes formas, não se restringindo a padrões modulares estéticos.

\section{Colaboradores}

JSGZ Witt fez busca de artigos erevisão da literatura, síntese das bibliografias e redação do texto e AP Schneider foi orientadora e idealizadora do tema, e fez a revisão final do trabalho. 


\section{Referências}

1. Russo R. Imagem corporal: construção através da cultura do belo. Movimento e Percepção 2005; 6(5):80-90.

2. Santos $M$, M eneguci $L, M$ endonça $A A F$. Padrão alimentar anormal em estudantes universitárias das áreas de nutrição, enfermagem e ciências biológicas. Ciên et Praxis 2008; 1(1):1-4.

3. Braga PD, Molina M CB, Cade NV. Expectativas de adolescentes em relação a mudanças do perfil nutricional. Cien Saude Colet 2007; 12(5):1221-1228.

4. Neto PP, Capari SNC. A medicalização da beleza. Interface (Botucatu) 2007; 11(23):569-584.

5. Serra GM A, Santos EM. Saúde e mídia na construção da obesidade e do corpo perfeito. Cien Saude Colet 2003; 8(3):691-701.

6. Almeida SS, Nascimento PC, Quaioti TCB. Quantidade e qualidade de produtos alimentícios anunciados na televisão brasileira. Rev S Pública 2002; 36(3):353-355.

7. Alcântra MLB. O corpo do brasileiro: estudos de estética e beleza. Rev Antropol 2001; 44(2):231-234.

8. Duke L. Get real: Cultural relevance and resistance to the mediated feminine ideal. Psychology and M arketing 2002; 19(2):211-235.

9. Souto S, Ferro-Bucher JSN. Práticas indiscriminadas de dietas de emagrecimento e o desenvolvimento de transtornos alimentares. Rev N utr 2006; 19(6):693-704

10. Branco LM, Hilário MOE, Cintra IP. Percepção e satisfação corporal em adolescentes e a relação com seu estado nutricional. Rev Psiq Clín 2006; 33(6): 292-296.

11. Groesz LM, Levine M P, Murnen SK. The effect of experimental presentation of thin media image on body satisfaction: a meta-analytic rewien. International Journal of Eating Disorders 2002; 31(1):1-16.

12. Gomes GR, Caramaschi S. Valorização de beleza e inteligência por adolescentes de diferentes classes sociais. Psicol Estud 2007; 12(2):295-303.

13. Thomsen SR, McCoy JK, Gustafson RL, Williams $M$. Motivations for reading beauty and fashion magazines and anorexic risk in college-age women. M edia Psychology 2002; 2(4):113-135.

14. Vasques F, Martins FC, Azevedo AP. Aspectos psiquiátricos no tratamento da obesidade. Rev Psiq Clín 2004; 31(40):195-198.

15. Kawalski M, Ferreira M BR. Estética, corpo e cultura. Rev Reflexões 2007; 5(2):90-113.

16. Bruck M, M elnyk L. Individual differences in children's sugestibility:a rewiew and synthesis. Applied Congnitive Psychology 2004; 18(8):947-996.

17. Saikali CJ, Soubhia CS, Scalfaro BM, Cordas TA. Imagem corporal nos transtornos alimentares. Rev Psiq Clín 2004; 31(4):164-166.

18. Goldenberg M. Gênero e corpo brasileiro. Psicol Clín 2005; 17(2):65-80.

19. Savoia M G. A imagem corporal. Rev Bras Psiquiatria 2003: 25(2):126

20. Almeida ACN, Carneiro CH, Araújo DR, Santos FP, Neto JMV, Marino MAP et al. Corpo, estética e obesidade: reflexões baseadas no paradigma da indústria cultura. Estudos 2006: 33(10):789-812.
21. Bosi M LM, Luiz RR, Morgado CMC, Cota MLS, Carvalho RJ. Autopercepção da imagem corporal entre estudantes do Rio de Janeiro. J Bras Psiquiatr 2006; 55(1):34-40.

22. Bosi MLM, Luiz RR, Uchimura KY, Oliveira FP. Comportamento alimentar e imagem corporal entres estudantes de educação física. J Bras Psiquiatr 2008; 57(1):28-33.

23. Gonçalves $T D$, Barbosa $M P$, Rosa $L C L$, Rodrigues AM. Comportamento anórexico e percepção corporal em universitários. J Bras Psiquiatr 2008; 57(3):166-170.

24. Poulain JP, Proença RPC. Reflexões metodológicas para o estudo das práticas alimentares. Rev Nutr 2003; 16(4):365-386.

25. Lanzillotti HS, Couto RM, Afonso FM. Pirâmides alimentares: uma leitura semiótica. Rev N utr 2005; 18(6):785-792.

26. Liberato SC, Pinheiro HM. Fortification of industrialized foods with vitamins. Rev Nutr 2006; 19(2):215-231

27. Rodrigues EM, Soares FPTP, Boog M CF. Resgate do conceito de aconselhamento no contexto do atendimento nutricional. Rev Nutr 2005; 18(1):119-128.

28. Borzekowski DL, Robinson TN. The 30-second effect: an experiment revealing the impact of television commercials on food preferences of preschoolers. J Am Diet Assoc 2001(1); 101:42-46.

29. Neutzling MB, Cora LPA, Vieira M FA, Hallal PC $M$ enezes AM B. Freqüência de consumo de dietas ricos em gordura e pobres em fibras entre adolescentes. Rev Saude Publica 2007; 41(3):336-342.

30. Santos LAS. Educação alimentar e nutricional no contexto da promoção de práticas alimentares saudáveis. Rev Nutr 2005; 18(5):681-692

31. Painter J, Rah JH, Lee YK. Comparison of international food guide pictorial representations. J Am Diet Assoc 2002; 102(4):483-189.

32. M ota Jf, Rinaldi AEM, Pereira AF, Maestá N, Scarpin M M, Bunini RC. Adaptação do índice de alimentação saudável ao guia alimentar da população brasileira. Rev Nutr 2008; 11(5):545-552.

33. Marcondelli P, Costa THM, Schmitz BAS. Physical activity level and food intake habits of university students fron 3 to 5 semester in the health area. Rev Nutr 2008; 21(1):39-47.

34. Rodrigues EM, Boog M CF. Problematização como estratégia de educação nutricional com adolescentes obesos. Cad Saude Publica 2006; 22(5):923-931.

35. Buss PM. Promoção da saúde e qualidade de vida. Cien Saude Colet 2000; 5(1):163-177.

36. Martins C. Aconselhamento Nutricional. In: Cuppari L. Guia de nutrição: nutrição clínica no adulto. São Paulo: Manole; 2005. p.129-145.

37. Duchesne M, Almeida PEM. Terapia cognitivocomportamental dos transtornos alimentares. Rev Bras Psiquiatria 2002; 24(3):49-53. 
38. Duchesne $M$, Appolinario JC, Rangé $B P$, Fandiño J, M oya T, Freitas SR. The use of a manual-drivem group cognitive behavior therapy in a Brazilian sample of obese individuals with binge-eating disorder. Rev Bras Psiquiatria 2007; 29(1):23-25.

39. Duschesne $M$, Appolinário JC, Rangé $B P$, Freitas $S$, Papelbaum M, Coutinho W. Evidências sobre a terapia cognitivo-comportamental no tratamento de obesos com transtorno da compulsão alimentar periódica. Rev Psiquiatr Rio Gd Sul 2007; 29(1):80-92.

40. Grauo S, Pereira EF, Lopes AS, Araújo VC, Legnani RFS, Borgatto AF. Associação entre a percepção da imagem corporal e indicadores antropométricos de escolares. Rev Bras Educ Fís Esp 2008; 22(2):129-138.

Artigo apresentado em 01/09/2008

Aprovado em 20/01/2009

Versão final apresentada em 05/02/2009 